\title{
Performance comparison of multi-domain routing schemes in GMPLS networks with BGP
}

\author{
Manolova, Anna Vasileva; Ruepp, Sarah Renée; Dittmann, Lars
}

Published in:

International Conference on Photonics in Switching, 2009. PS '09

Link to article, DOI:

10.1109/PS.2009.5307776

Publication date:

2009

Document Version

Publisher's PDF, also known as Version of record

Link back to DTU Orbit

Citation (APA):

Manolova, A. V., Ruepp, S. R., \& Dittmann, L. (2009). Performance comparison of multi-domain routing schemes in GMPLS networks with BGP. In International Conference on Photonics in Switching, 2009. PS '09 (pp. 1-2). IEEE. https://doi.org/10.1109/PS.2009.5307776

\section{General rights}

Copyright and moral rights for the publications made accessible in the public portal are retained by the authors and/or other copyright owners and it is a condition of accessing publications that users recognise and abide by the legal requirements associated with these rights.

- Users may download and print one copy of any publication from the public portal for the purpose of private study or research.

- You may not further distribute the material or use it for any profit-making activity or commercial gain

- You may freely distribute the URL identifying the publication in the public portal 


\title{
Performance Comparison of Multi-Domain Routing Schemes in GMPLS Networks with BGP
}

\author{
A.V. Manolova, S. Ruepp, L. Dittmann \\ Network Technologies and Service Platforms, DTU Fotonik, Technical University of Denmark, Kgs. Lyngby, Denmark \\ \{anva, srru, ladit\}@fotonik.dtu.dk
}

\begin{abstract}
We compare an enhancement of the BGP protocol for TE support in GMPLS networks with a simple TE extension of BGP in terms of signaling overhead and connection blocking ratio. Our results show increased performance of the enhanced BGP.
\end{abstract}

Keywords: GMPLS, BGP, multi-domain routing

\section{Introduction}

Providing Quality of Service (QoS) across multiple domains in WDM transport networks is a relatively new area of research. After the proven success of the Generalized Multi-Protocol Label Switching (GMPLS) architecture [1] to provide Traffic Engineering (TE) and Quality of Service (QoS) support within a single domain the next step is extending its functionality across domains. Thus, IETF is currently developing the Path Computation Element (PCE) architecture [2], which together with the existing GMPLS protocols can provide QoS compliant Label Switched Paths (LSPs) across multiple domains. The PCE though is not the only option for routing in multi-domain connection-oriented networks. Much work has been done to modify and extend the existing Internet multi-domain routing protocol - the Border Gateway Protocol (BGP) [3] for GMPLS networks. Extensions for multi-path dissemination [4], QoS support [5] as well as Optical BGP [6] have been proposed.

One of the recent works on the topic is from [7], where the authors propose an enhanced BGP protocol, fully compliant with the GMPLS framework, for establishing LSPs across multiple Autonomous Systems (ASes). In this paper we investigate the efficiency of the proposed Enhanced BGP from two perspectives. First, with investigate the signaling overhead during BGP convergence when the TE state of paths is updated. Second, we compare the performance of the suggested protocol with a simple version of QoS-enhanced BGP when limited wavelength conversion is applied in the network.

\section{Modified BGP for multi-domain routing in connection-oriented networks}

\subsection{TE extensions for BGP}

BGP is a path vector protocol which disseminates network reachability information across AS borders. For scalability reasons the protocol carries as little information as possible - namely the reachable destination, the next hop to reach it and a list of ASes to be traversed on the way. No TE information is included, which obstructs the extension of the TE principles across multiple ASes. There are options for applying TE principles with BGP but they have mainly local application (between pair of domains), do not cross multiple ASes and do not meet the requirements of GMPLS networks. The lack of TE information distribution is not the only problem with BGP [7]. For GMPLS networks features such as multiple paths per destination for resilience support, source routing for higher control of the head-end of the connection over the provisioning process, as well as fast recovery from failures must be supported. BGP does not provide such features. Only one path is disseminated per destination, and there is hence a strong path dependency between domains which results in slow protocol convergence after network state change. Solutions to some of these problems can be found in $[4,5,6]$.

In our previous work [7] we design an Enhanced BGP which faces all outlined problems. The proposal disseminates multiple paths per destination, provides TE information per path and is fully compatible with the GMPLS signaling procedures for LSP setup. In this work we investigate the performance of the suggested enhancement and compare it to a QoS-enhanced BGP [5] supported with limited wavelength conversion.

\subsection{TE connection provisioning with Enhanced BGP}

The main advantage of the Enhanced BGP protocol is the dissemination of multiple paths per destination, which each has an end-to-end TE metric, based on which path is chosen at the time of LSP request. Unlike the standard BGP, the Enhanced BGP does not perform path selection under the BGP operation, but only path dissemination. Figure 1 illustrates the routing process. Furthermore, the TE state of the paths is updated at regular intervals. Results for the relation between the updating interval and the LSP blocking ratio can be found in [7].

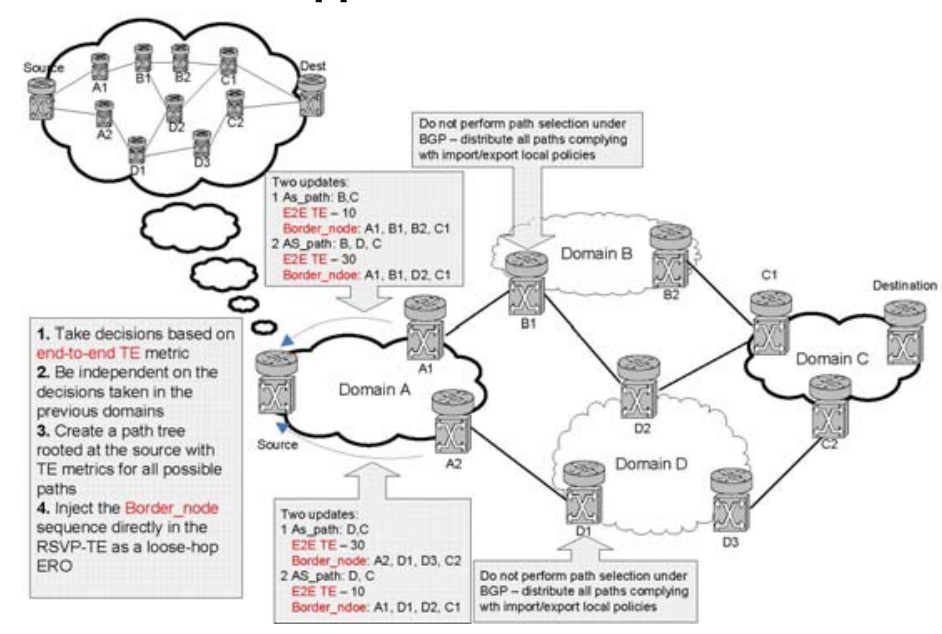

Figure 1: Path dissemination and selection with Enhanced BGP 


\section{Simulation scenario and results}

We are using the discrete event simulation tool OPNET Modeler [8], to model the behavior of the Enhanced BGP protocol and compare it with a QoS-BGP implementation [5], where a TE metric (minimum available wavelengths) is disseminated and is considered as first decision criterion under the BGP path selection process. As a test instance, we use the COST 266 [9] Pan-European network. The intra-domain topologies of the separate domains are randomly generated, and have between one and four source/destination nodes. There are 46 source/destination pairs in total, 22 domains and 42 inter-domain links. Each link has 30 wavelengths. For the Enhanced BGP routing no wavelength conversion is applied. Traffic is uniformly distributed between the source/destination pairs. First Fit wavelength assignment is applied using RSVP-TE signaling for resource reservation.

The first performance measure we evaluate is the signaling overhead (number of UPDATE messages) of the protocol needed to update the TE state of the used paths in the network. Figure 2 presents the result. The BGP-TE protocol results in twice as much overhead compared to the Enhanced BGP, which is due to the path dependency problem of the standard BGP operation [3]. Moreover, the Enhanced BGP overhead is independent on the traffic load in the network whereas the BGP-TE results in unstable amount of needed UPDATE messages.

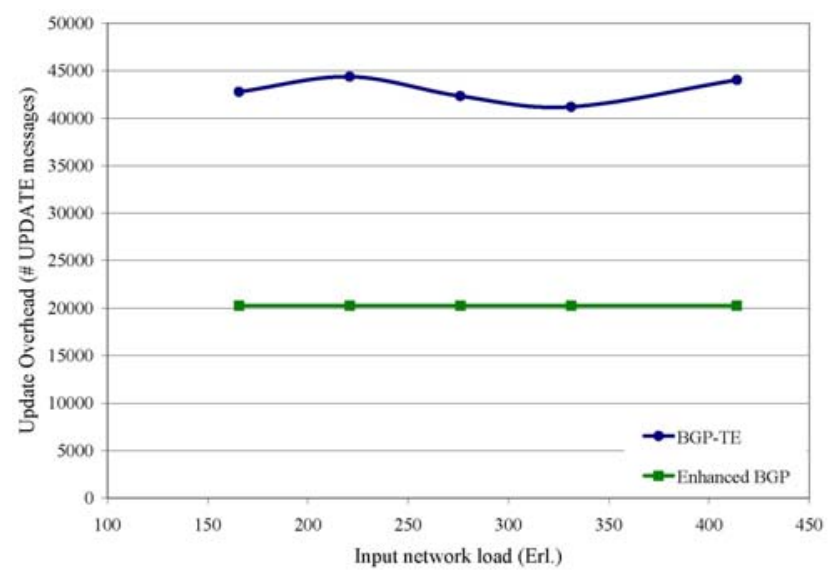

Figure 2: Update overhead

The second performance measure we investigate is the blocking ratio for LSP requests in the network when limited wavelength conversion is applied for the BGP-TE scheme. From figure 3 it can be seen that without wavelength converters in the network the Enhanced BGP significantly outperforms the BGP-TE solution for all tested load ranges. When the number of used wavelength converters increases for the BGP-TE scheme, its performance improves but even at 10 wavelength converters per node the Enhanced BGP outperforms the BGP-TE solution for the low load ranges (approximately $22 \%$ link utilization).

\section{Conclusion}

In this paper we compare the performance of a recently suggested Enhanced BGP protocol for TE in multi-domain GMPLS networks with a simple TE extension of the standard
BGP protocol. Our simulation results show significant decrease in the needed amount of signaling information for updating the TE state of paths in the network. Independence of the traffic load in the network is also observed, which is beneficial for easy to plan and maintain control plane. Furthermore, we show that the Enhanced BGP can provide lower blocking probability compared to the BGP-TE even if limited wavelength conversion is applied under the BGP-TE routing. These results illustrate that the Enhanced BGP protocol is a promising solution for routing in the next generation dynamic GMPLS networks.

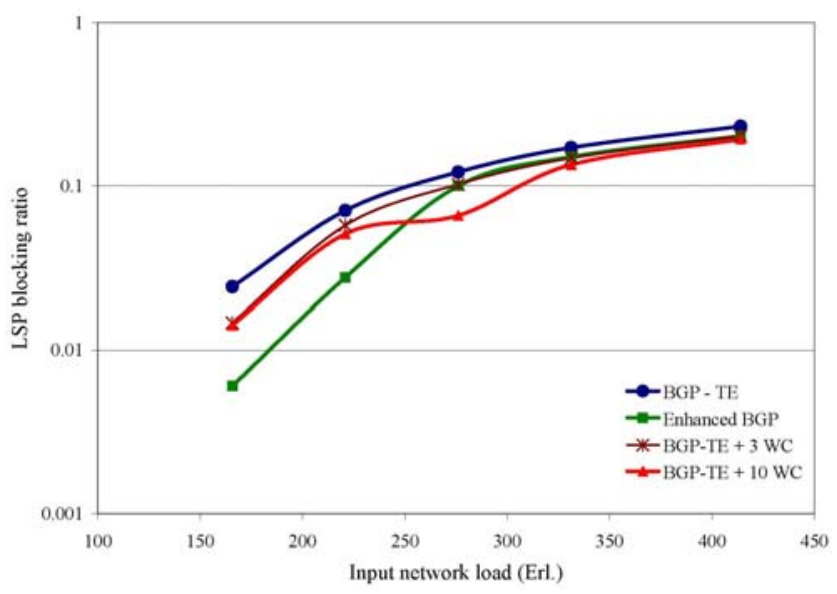

Figure 3: LSP blocking ratio for two routing schemes with and without wavelength conversion

\section{Acknowledgment}

The work described in this paper was carried out with the support of the BONE-project ("Building the Future Optical Network in Europe"), a Network of Excellence funded by the European Commission through the 7th ICT-Framework Programme.

\section{References}

[1] Mannie, E.: "Generalized Multi-Protocol Label Switching (GMPLS) Architecture", RFC 3945, October 2004.

[2] Farrel, A., Vasseur, JP., and Ash. J.: "A Path Computation Element (PCE) - Based Architecture", RFC 4655 (Informational), August 2006.

[3] Rekhter, Y., and Hares, S.: "A Border Gateway Protocol 4 (BGP-4)", RFC 4271, January 2006.

[4] Kushman, N., Kandula, S., Katabi, D., and Maggs. B.: "R-BGP: Staying Connected in a Connected World", 4th USENIX Symposium on Networked Systems Design \& Implementation, April 2007.

[5] Cristallo, G., Jacquenet, C.: "Providing Quality of Service Indication by the BGP-4 Protocol: the QOS_NLRI attribute" IETF draft, Exp. 2003, draft-jacquenet-qos-nlri-05.txt.

[6] M. $\sim$ Blanchet, F. Parent, B. St-arnaud: "Optical BGP (OBGP): InterAS Lightpath Provisioning", IETF draft, Exp. 2001, ietf-draft-parent-obgp-01.txt.

[7] Manolova, A., Ruepp, S., Buron, J., Dittmann. L.: "On the Efficiency of BGP-TE Extensions for GMPLS Multi-Domain Routing", $13^{\text {th }}$ ONDM Conference, February 2009.

[8] OPNET Technologies, Inc. www.opnet.com

[9] R. Inkret et al. "Advanced Infrastructure for Photonic Networks", Extended final report of COST Action 266, available at http://www.ufe.cz/dpt240/cost266/. 